\title{
REMARKS ON WEAK STABILIZATION OF SEMILINEAR WAVE EQUATIONS
}

\author{
Alain Haraux ${ }^{1}$
}

\begin{abstract}
If a second order semilinear conservative equation with esssentially oscillatory solutions such as the wave equation is perturbed by a possibly non monotone damping term which is effective in a non negligible sub-region for at least one sign of the velocity, all solutions of the perturbed system converge weakly to 0 as time tends to infinity. We present here a simple and natural method of proof of this kind of property, implying as a consequence some recent very general results of Judith Vancostenoble.
\end{abstract}

Mathematics Subject Classification. 35B35, 35L55, 35L90.

Received June 19, 2000. Revised November 11, 2000 and May 4, 2001.

\section{INTRODUCTION}

Following a recent work of Vancostenoble [20], we investigate the weak stabilization to 0 of solutions to the equations

$$
\begin{gathered}
u_{t t}+A u+Q\left(u_{t}\right)=0 \quad \text { on } \\
u_{t t}+A u+g(u)+Q\left(u_{t}\right)=0 \quad \text { on }
\end{gathered}
$$

where $A$ is a linear positive selfadjoint operator of elliptic type on $H=L^{2}(\Omega), \Omega$ is a bounded open domain of $\mathbb{R}^{N}$, the term $-Q\left(u_{t}\right)$ represents a possibly non monotone feedback dissipation acting on a "non negligible" part $Y$ of $\bar{\Omega}$ and $g(u)$ stands for the Nemytsckii operator associated to some numerical function $g \in C^{1}(\mathbb{R})$. Concerning (1.1), the original proof from [20] was inspired both by the work of Slemrod [19] and the techniques of Conrad and Pierre [10]; here we present a new simplified proof relying on almost periodicity of generalized solutions to

$$
u_{t t}+A u=0 \quad \text { on } \quad \mathbb{R}
$$

which implies some essential oscillatory behavior of those solutions on $\mathbb{R} \times Y$. Weak convergence is proved, following the philosophy introduced in [11] (cf. also [12,13]) under the hypothesis that the damping is effective at least for one sign of the velocity (one-sided dissipation). This method is applicable to more complicated problems of the form (1.2) when solutions of

$$
u_{t t}+A u+g(u)=0 \quad \text { on } \quad \mathbb{R}
$$

are known to be oscillatory on $\mathbb{R} \times Y$. A typical example is the nonlinear string equation

$$
u_{t t}-u_{x x}+g(u)+a(x) q\left(u_{t}\right)=0 \quad \text { on } \quad \mathbb{R}^{+} \times \Omega
$$

Keywords and phrases: Weak stabilization, semilinear, wave equations.

1 Université Pierre et Marie Curie, Analyse Numérique, Tour 55-65 5 étage, 4 place Jussieu, 75252 Paris Cedex 05, France; e-mail: haraux@ann.jussieu.fr 
with homogeneous Dirichlet boundary conditions on $\partial \Omega$ with $\Omega=(0, L)$ when $g$ is odd nonincreasing, $a \geq 0$, $a>0$ on an open subdomain $\omega$ and $q$ satisfies:

$$
q \in C^{1}, \quad q(v) v \geq 0 \quad \text { on } \quad \mathbb{R}, \quad q(v)>0 \quad \text { for all } \quad v>0 .
$$

Here even if $q$ is monotone, compactness of trajectories in the energy space is not known. The consideration of more general similar examples sheds a new light on the interest of oscillatory behavior of semilinear conservative systems.

\section{INTERNAL DAMPING}

In this section, we consider the case of equation (1.1) with internal damping, which means that $Y=\omega$, an open subset of $\Omega$. In other terms we consider the equation

$$
u_{t t}+A u+a(x) q\left(u_{t}\right)=0 \quad \text { on } \quad \mathbb{R}^{+}
$$

where $a \in L^{\infty}(\Omega), a \geq 0$ a.e. in $\Omega$ and $a \geq \eta>0$ a.e. in $\omega$. The function $q \in C(\mathbb{R})$ satisfies

$$
q(v) v \geq 0 \quad \text { on } \quad \mathbb{R}, \quad q(v)>0 \text { for all } \quad v>0 .
$$

We consider a Hilbert space $V \subset H=L^{2}(\Omega)$ with compact and dense imbedding. The linear operator $A: V \rightarrow$ $V^{\prime}$ satisfies the following conditions

$$
A \in \mathcal{L}\left(V, V^{\prime}\right) ; \quad \forall v \in V,<A v, v>\geq \alpha\|v\|^{2}
$$

where $\alpha>0$ and $\|v\|$ denotes the norm of $v$ in $V$. Assume that

$$
W=L^{\infty}(\Omega) \cap V \quad \text { is dense in } \quad V .
$$

We say that a function $u: \mathbb{R}^{+} \rightarrow V$ is a solution of (2.1) if $u$ satisfies the following conditions:

$$
\begin{gathered}
u \in C\left(\mathbb{R}^{+}, V\right) \cap C^{1}\left(\mathbb{R}^{+}, H\right) \cap W_{\mathrm{loc}}^{2,1}\left(\mathbb{R}^{+}, V^{\prime}\right), \quad a(x) q\left(u^{\prime}\right) \in L_{\mathrm{loc}}^{1}\left(\mathbb{R}^{+}, L^{1}(\Omega)\right) \\
\forall \varphi \in W, \quad<u ”(t)+A u(t)+a(x) q\left(u^{\prime}(t)\right), \varphi>=0 \quad \text { a.e. on } \mathbb{R}^{+} .
\end{gathered}
$$

In addition, we say that $u$ satisfies the energy inequality if

$$
\forall T>0, \quad E(T)+\int_{0}^{T} \int_{\Omega} a(x) q\left(u^{\prime}(t, x)\right) u^{\prime}(t, x) \mathrm{d} x \mathrm{~d} t \leq E(0)
$$

with

$$
\forall t \geq 0, \quad E(t):=\frac{1}{2}\left\{\left|u^{\prime}\right|^{2}(t)+<A u(t), u(t)>\right\} .
$$

Finally we say that unique determination of eigenfunctions of A holds in $\omega$ if

$$
\forall \lambda>0, \forall \varphi \in V, \quad A \varphi=\lambda \varphi \quad \text { and } \quad \varphi \equiv 0 \quad \text { in } \omega \quad \Longrightarrow \quad \varphi \equiv 0 \quad \text { in } \Omega
$$

The main result of this section is:

Theorem 2.1. Under the above hypotheses, let $u$ be a solution of (2.1) satisfying the energy inequality and assume that unique determination of eigenfunctions of $A$ holds in $\omega$. Then as $t \rightarrow \infty$ :

$$
\left(u(t), u_{t}(t)\right) \rightarrow(0,0) \quad \text { in } V \times H .
$$


Proof. Let $t_{n}$ be a sequence of positive real numbers tending to $+\infty$ with $n$ and $u_{n}(t, x)=u\left(t+t_{n}, x\right)$ for all $(t, x) \in\left[-t_{n},+\infty\right) \times \Omega$. Given any $\tau>0$, the function $u_{n}(t, x)$ is well defined a.e. on $\Omega$ as an element of $V$ for all $t \in[-\tau, \tau]=: J_{\tau}$ as soon as $t_{n} \geq \tau$. In addition it follows easily from the energy inequality that $u_{n}$ is bounded uniformly in $C\left(J_{\tau}, V\right) \cap C^{1}\left(J_{\tau}, H\right)$ for $n \geq \tau$. In particular, by Ascoli-Arzela's theorem, we can assume that a certain subsequence $u_{n_{k}}=: z_{k}$ converges in $C\left(J_{\tau}, H\right)$ for all $\tau>0$, to a certain limiting function $z \in C(\mathbb{R}, H)$. Moreover $z$ is bounded in $H$ and weakly differentiable $\mathbb{R} \rightarrow H$ with bounded derivative. From the energy inequality it also follows, by using continuity of $q$ at 0 , that

$$
\forall \tau>0, \quad a(x) q\left(u_{n}^{\prime}(t, x)\right) \rightarrow 0 \quad \text { in } \quad L^{1}\left(J_{\tau} \times \Omega\right) \quad \text { as } \quad n \rightarrow \infty
$$

for all $\tau>0$. By using as test functions the eigenfunctions of $A$, it follows easily that $z$ is in fact a solution of

$$
z \in C(\mathbb{R}, V) \cap C^{1}(\mathbb{R}, H) \cap C^{2}\left(\mathbb{R}, V^{\prime}\right), \quad z "+A z=0 .
$$

In particular, $z$ is a $C^{1}$ almost periodic vector function: $\mathbb{R} \rightarrow H, c f$. e.g. $[1,3,16]$. From $(2.2)$ we infer that in fact

$$
z^{\prime}=z_{t} \leq 0 \quad \text { a.e. on } \quad \mathbb{R} \times \omega .
$$

Assuming (2.3) the conclusion follows easily. Indeed then the trace of $z$ on $\omega$ is a non-increasing function: $\mathbb{R} \rightarrow L^{2}(\omega)$. Classically, such a function has to remain constant with respect to $t$ for almost all $x \in \omega$ (this can be checked easily on multiplying by any smooth nonnegative function supported in $\omega$ and applying a classical recurrence property of real-valued almost periodic vector function, cf. e.g. [1,3] or Cor. 4.2.6, p. 50 of [16], or even Cor. I.3.1.6 of [15]), therefore if we consider ( $c f$. e.g. $[1,18])$ the Fourier-Bohr expansion of $z$ given by the formula

$$
z(t, x)=\sum_{n=1}^{\infty}\left[\varphi_{n}(x) \cos \left(t \sqrt{\lambda_{n}}\right)+\psi_{n}(x) \sin \left(t \sqrt{\lambda_{n}}\right)\right]
$$

where $\left\{\lambda_{n}\right\}_{n \geq 1}$ is the increasing sequence of eigenvalues of $A$ and

$$
\begin{aligned}
& \varphi_{n}(x)=\lim _{T \rightarrow \infty} \frac{1}{T} \int_{-T}^{T} \cos \left(t \sqrt{\lambda_{n}}\right) z(t, x) \mathrm{d} x \\
& \psi_{n}(x)=\lim _{T \rightarrow \infty} \frac{1}{T} \int_{-T}^{T} \sin \left(t \sqrt{\lambda_{n}}\right) z(t, x) \mathrm{d} x
\end{aligned}
$$

the functions $\varphi_{n}(x)$ and $\psi_{n}(x)$ are eigenfunctions of $A$ which vanish in $\omega$. By the unique determination of eigenfunctions of $A$ in $\omega$ the result follows at once. Now (2.3) will follow as an easy consequence of the following

Lemma 2.2. Let $(U, \mu)$ be any finitely measured space and $w_{n} \in L^{p}(U, \mathrm{~d} \mu)$ with $p>1$. Assume

Then we have:

$$
\begin{gathered}
w_{n} \rightarrow w \quad \text { in } L^{p}(U, \mathrm{~d} \mu) \quad \text { as } n \rightarrow \infty \\
\mu\left\{w_{n} \geq 0\right\} \rightarrow 0 \quad \text { as } \quad n \rightarrow \infty
\end{gathered}
$$

$$
\mu\{w>0\}=0
$$

Proof of Lemma 2.2. Let $y_{n}=\inf \left\{w_{n}, 0\right\}=-w_{n}^{-} \leq 0$. We have

$$
\left\|y_{n}-w_{n}\right\|_{L^{1}(U)} \leq\left\|w_{n}\right\|_{L^{p}(U)}\left[\mu\left\{w_{n} \geq 0\right\}\right]^{1-\frac{1}{p}} \rightarrow 0 \quad \text { as } \quad n \rightarrow \infty .
$$

In particular we have

$$
y_{n} \rightarrow w \quad \text { in } L^{1}(U, \mathrm{~d} \mu) \quad \text { as } \quad n \rightarrow \infty
$$


Since by construction, $y_{n} \leq 0, \quad$ p.a.e. on $\quad U, \quad(2.6)$ follows immediately.

End of proof of Theorem 2.1. From (2.2) we finally deduce (2.3) as follows. Let $\tau>0$ be fixed and set $U=J_{\tau} \times \omega$ and denote by $\mu$ the Lebesgue measure on $U$ in $\mathbb{R}^{N+1}$. We establish that $w=z^{\prime} \leq 0$, a.e. on $U$. In order to do that it is sufficient to establish, for any given $\varepsilon>0$, the inequality $w=z^{\prime} \leq \varepsilon, \mu-$ a.e. on $U$. First, given any $\delta>0$ we select $M=M(\delta)$ such that

$$
\forall n \geq \tau, \quad \mu\left\{(t, x) \in U, z_{n}^{\prime}(t, x) \geq M\right\} \leq \delta .
$$

This is made possible by boundedness of $u^{\prime}$ in $L^{2}(\Omega)$. In particular we have

$$
\forall n \geq \tau, \quad \mu\left\{(t, x) \in U, z_{n}^{\prime}(t, x) \geq \varepsilon\right\} \leq \delta+\mu\left\{(t, x) \in U, \varepsilon \leq z_{n}^{\prime}(t, x) \leq M\right\} .
$$

As a consequence of $(2.2)$ and by compactness of $[\varepsilon, M]$ it now follows easily from the properties $a \geq \eta>0$ a.e. in $\omega$ and $a(x) q\left(u_{n}^{\prime}(t, x)\right) \rightarrow 0$ in $L^{1}(U)$ as $n \rightarrow \infty$, that

$$
\lim _{n \rightarrow \infty} \mu\left\{(t, x) \in U, \varepsilon \leq z_{n}^{\prime}(t, x) \leq M\right\}=0 .
$$

Therefore

$$
\limsup _{n \rightarrow \infty} \mu\left\{(t, x) \in U, z_{n}^{\prime}(t, x) \geq \varepsilon\right\} \leq \delta .
$$

Since $\delta>0$ is arbitrary, this means

$$
\lim _{n \rightarrow \infty} \mu\left\{(t, x) \in U, z_{n}^{\prime}(t, x) \geq \varepsilon\right\}=0 .
$$

By Lemma 2.2 applied with $w_{n}=z_{n}^{\prime}-\varepsilon$ we deduce $z^{\prime} \leq \varepsilon, \quad \mu-$ a.e. on $U$. The proof is now complete.

\section{THE GENERAL CASE}

In this section, we consider the case of equation (1.1) with a damping possibly distributed on a lower dimensional subset. For instance $Y$ can be a relatively open subset of $\partial \Omega$, in which case (1.1) can take the form of a wave equation with boundary dissipation

$$
u_{t t}-\Delta u=0 \quad \text { on } \quad \mathbb{R}^{+} \times \Omega ; \quad \frac{\partial u(t, x)}{\partial \nu}+a(x) q\left(u_{t}\right)=0 \quad \text { on } \quad \mathbb{R}^{+} \times \partial \Omega
$$

considered in [21] by Vancostenoble.

In the general case we consider a function $q \in C(\mathbb{R})$ satisfying $(2.2)$ and the stronger condition

$$
\forall \varepsilon>0, \quad \inf _{s \geq \varepsilon} q(s)>0 .
$$

We consider a Hilbert space $V \subset H=L^{2}(\Omega)$ with compact and dense imbedding. The linear operator $A: V \rightarrow$ $V^{\prime}$ satisfies the following conditions

$$
A \in \mathcal{L}\left(V, V^{\prime}\right) ; \quad \forall v \in V,<A v, v>\geq \alpha\|v\|^{2}
$$

where $\alpha>0$ and $\|v\|$ denotes the norm of $v$ in $V$. Assume that

$$
W=C(\bar{\Omega}) \cap V \quad \text { is dense in } \quad V .
$$


In addition we consider a compact subset $Y$ of $\bar{\Omega}$ and a nonegative bounded measure $\mu \in M_{B}(Y)$. We say that a function $u: \mathbb{R}^{+} \rightarrow V$ is a solution of (1.1) if $\mathrm{u}$ satisfies the following conditions:

$$
\begin{gathered}
u \in C\left(\mathbb{R}^{+}, V\right) \cap C^{1}\left(\mathbb{R}^{+}, H\right) \cap W_{\mathrm{loc}}^{2,1}\left(\mathbb{R}^{+}, V^{\prime}\right) \\
a(y) q\left(u^{\prime}(t, y)\right) \in L_{\mathrm{loc}}^{1}\left(\mathbb{R}^{+}, L^{1}(Y, \mathrm{~d} \mu)\right) \\
\forall \varphi \in W, \quad\left\langle u^{\prime \prime}(t)+A u(t), \varphi\right\rangle+\int_{Y} a(y) q\left(u^{\prime}(t, y)\right) \varphi(y) \mathrm{d} \mu(y)=0 \quad \text { a.e. on } \quad \mathbb{R}^{+} .
\end{gathered}
$$

In addition, we say that $\mathrm{u}$ satisfies the energy inequality if

$$
\forall T>0, \quad E(T)+\int_{0}^{T} \int_{Y} a(y) q\left(u^{\prime}(t, y)\right) u^{\prime}(t, y) \mathrm{d} \mu(y) \mathrm{d} t \leq E(0)
$$

with

$$
\forall t \geq 0, \quad E(t):=\frac{1}{2}\left\{\left|u^{\prime}\right|^{2}(t)+\langle A u(t), u(t)\rangle\right\}
$$

Finally we say that unique determination of eigenfunctions of $A$ holds in $\omega \subset Y$ if

$$
\forall \lambda>0, \forall \varphi \in V, \quad A \varphi=\lambda \varphi \text { and } \varphi \equiv 0 \quad \mu \text {-a.e. in } \omega \Rightarrow \varphi \equiv 0 \quad \text { in } \Omega \text {. }
$$

The main result of this section is:

Theorem 3.1. Under the above hypotheses, assume that unique determination of eigenfunctions of $A$ holds in $\omega$ with $\inf _{y \in \omega} a(y)>0$. In addition assume that the trace $z \longrightarrow z \mid Y$ is well defined and continuous: $V \longrightarrow L^{1}(Y, \mathrm{~d} \mu)$. Let $u$ be a solution of (1.1) satisfying the energy inequality. Then as $t \rightarrow \infty$ :

$$
\left(u(t), u_{t}(t)\right) \rightarrow(0,0) \quad \text { in } V \times H .
$$

Proof of Theorem 3.1. Let $t_{n}$ be a sequence of positive real numbers tending to $+\infty$ with $n$ and $u_{n}(t, x)=$ $u\left(t+t_{n}, x\right)$ for all $(t, x) \in\left[-t_{n},+\infty\right) \times \Omega$. Keeping the notation of Section 2, by Ascoli-Arzela's theorem, we can assume that a certain subsequence $u_{n_{k}}=: z_{k}$ converges in $C\left(J_{\tau}, H\right)$ for all $\tau>0$, to a limiting function $z \in C(\mathbb{R}, H)$. Moreover $z$ is bounded in $H$ and weakly differentiable $\mathbb{R} \rightarrow H$ with bounded derivative. From the energy inequality it also follows, by using continuity of $q$ at 0 , that

$$
\forall \tau>0, \quad a(y) q\left(u_{n}^{\prime}(t, y)\right) \rightarrow 0 \quad \text { in } \quad L^{1}\left(J_{\tau} \times Y\right) \quad \text { as } \quad n \rightarrow \infty
$$

By using as test functions the eigenfunctions of $A$, it follows easily that $z$ is in fact a solution of

$$
z \in C(\mathbb{R}, V) \cap C^{1}(\mathbb{R}, H) \cap C^{2}\left(\mathbb{R}, V^{\prime}\right), \quad z^{\prime}+A z=0 .
$$

In particular, $z$ is a $C^{1}$ almost periodic vector function: $\mathbb{R} \rightarrow H$. However in the general case the analog of (2.3) is more delicate to establish and in fact, in order to use the trace operator: $V \longrightarrow L^{1}(Y, \mathrm{~d} \mu)$ we shall rely on a smoothing procedure replacing $u_{n_{k}}=: z_{k}$ by some auxiliary functions which have bounded time-derivatives in $V$. For any $\delta>0$, we consider

$$
u_{\delta}(t):=\int_{t}^{t+\delta} u(s) \mathrm{d} s
$$

and we define accordingly $u_{\delta, n}(t)$ and $z_{\delta}(t)$. From (2.2) and (3.2) we infer that in fact

$$
z_{\delta}^{\prime}(t, y) \leq 0 \quad \mu \text {-a.e. on } \quad \mathbb{R} \times \omega .
$$


In order to establish (3.3), first of all from the energy inequality we deduce

$$
\int_{-\tau}^{\tau} \int_{\omega}\left(u_{n}^{\prime}-\varepsilon\right)^{+}(t, y) \mathrm{d} \mu(y) \mathrm{d} t \rightarrow 0
$$

valid for all $\varepsilon>0$. On the other hand we have for each $\delta \in(0, \tau)$

$$
u_{\delta, n}^{\prime}(t, y)-\delta \varepsilon \leq \int_{t}^{t+\delta}\left(u_{n}^{\prime}-\varepsilon\right)^{+}(t, y) \mathrm{d} s
$$

almost-everywhere on $\Omega$ and in particular for any nonnegative function $\zeta \in L^{\infty}(\omega, \mathrm{d} \mu)$ we find, since $\varepsilon$ is arbitrarily small

$$
\forall t \in \mathbb{R}, \quad \limsup _{n \rightarrow \infty} \int_{\omega}\left(u_{\delta, n}^{\prime}(t, y) \zeta(y) \mathrm{d} \mu(y) \mathrm{d} t \leq 0 .\right.
$$

Now since $u_{\delta, n}^{\prime}(t, x)=u_{n}(t+\delta, x)-u_{n}(t, x)$, the convergence of to $z(t,$.$) in V$ weak implies the convergence pointwise in $t$ of $u_{\delta, n}^{\prime}(t,$.$) to z_{\delta}^{\prime}(t,$.$) in V$ weak. Since $V$ is a Hilbert space, there is, for each given $t$, a convex combination of the functions $u_{\delta, n}^{\prime}(t,$.$) which converges in fact to z_{\delta}^{\prime}(t,$.$) in V$ strong. By continuity of the trace: $V \rightarrow L^{1}(Y, \mathrm{~d} \mu)$ we obtain (3.3), more precisely we find

$$
\forall t \in \mathbb{R}, \quad \forall \zeta \in L_{+}^{\infty}(\omega, \mathrm{d} \mu), \quad \int_{\omega} z_{\delta}^{\prime}(t, y) \zeta(y) \mathrm{d} \mu(y) \mathrm{d} t \leq 0 .
$$

Now the conclusion follows easily. Indeed then the trace of $z_{\delta}$ on $\omega$ is a non-increasing almost periodic function: $\mathbb{R} \rightarrow L^{1}(\omega, \mathrm{d} \mu)$ which is also the trace of a solution of the linear equation. Classically, such a function has to remain constant, and by the unique determination of eigenfunctions of $\mathrm{A}$ in $\omega$, reasoning as in the proof of Theorem 2.1, we find that $z_{\delta}=0$ for all $\delta>0$. By letting $\delta \rightarrow 0$ we obtain $z=0$. Since the result is valid for any convergent subsequence of $\left(u_{n}, u_{n}^{\prime}\right)$ we conclude easily.

\section{Additional Results And Remarks}

The method of proof of Theorems 2.1 and 3.1 is applicable to more complicated problems of the form (1.2) when solutions of

$$
u_{t t}+A u+g(u)=0 \quad \text { on } \quad \mathbb{R}
$$

are known to be oscillatory on $\mathbb{R} \times Y$. As a typical example we consider the nonlinear string equation

$$
u_{t t}-u_{x x}+g(u)+a(x) q\left(u_{t}\right)=0 \quad \text { on } \quad \mathbb{R}^{+} \times(0, L) ; \quad u(t, 0)=u(t, L) \quad \text { on } \quad \mathbb{R}^{+}
$$

when $g$ is odd nonincreasing, $a \in L^{\infty}(0, L), \quad a \geq 0, \quad a(x) \geq \alpha>0$ on some open subdomain $\omega$ and $q$ satisfies:

$$
q \in C^{1}, \quad q(v) v \geq 0 \quad \text { on } \quad \mathbb{R}, \quad q(v)>0 \quad \text { for all } \quad v>0 .
$$

Here we obtain:

Theorem 4.1. Under the above hypotheses, let u be a solution of (4.1) satisfying the energy inequality

$$
\forall T>0, \quad E(T)+\int_{0}^{T} \int_{0}^{L} a(x) q\left(u_{t}(t, x)\right) u_{t}(t, x) \mathrm{d} x \mathrm{~d} t \leq E(0)
$$

with

$$
\forall t \geq 0, \quad E(t):=\frac{1}{2} \int_{0}^{L}\left\{u_{t}^{2}(t, x)+u_{x}^{2}(t, x)\right\} \mathrm{d} x+\int_{0}^{L} G(u(t, x) \mathrm{d} x
$$


where

Then as $t \rightarrow \infty$ :

$$
G(r):=\int_{0}^{r} g(s) \mathrm{d} s
$$

with

$$
\left(u(t), u_{t}(t)\right) \rightarrow(0,0) \quad \text { in } V \times H
$$

$$
V=H_{0}^{1}(0, L) \quad \text { and } \quad H=L^{2}(0, L)
$$

Proof of Theorem 4.1. Let $\Omega=(0, L)$, let $t_{n}$ be a sequence of positive real numbers tending to $+\infty$ with $n$ and $u_{n}(t, x)=u\left(t+t_{n}, x\right)$ for all $(t, x) \in\left[-t_{n},+\infty\right) \times \Omega$. Keeping the notation of Section 2, we obtain that a certain subsequence $u_{n_{k}}=: z_{k}$ converges in $C\left(J_{\tau}, H\right)$ for all $\tau>0$, to a certain limiting function $z \in C(\mathbb{R}, H)$. From the energy inequality it also follows, by using continuity of $q$ at 0 , that

$$
\forall \tau>0, \quad a(x) q\left(u_{n}^{\prime}(t, x)\right) \rightarrow 0 \quad \text { in } \quad L^{1}\left(J_{\tau} \times \Omega\right) \quad \text { as } \quad n \rightarrow \infty .
$$

It follows easily that $z$ is in fact a solution of

with in addition

$$
\begin{aligned}
& z \in C(\mathbb{R}, V) \cap C^{1}(\mathbb{R}, H) \cap C^{2}\left(\mathbb{R}, V^{\prime}\right), \\
& z_{t t}-z_{x x}+g(z)=0 \quad \text { on } \quad \mathbb{R} \times(0, L)
\end{aligned}
$$

$$
z^{\prime}=z_{t} \leq 0 \quad \text { a.e. on } \quad \mathbb{R} \times \omega .
$$

As a consequence of $[2,3]$, it follows that $z=0$. The conclusion then follows easily from the fact that any sequence $\left(u\left(t_{n}\right), u^{\prime}\left(t_{n}\right)\right)$ has a subsequence converging weakly to $(0,0)$.

Remark 4.2. Here even if $q$ is monotone, compactness of trajectories in the energy space is not known.

Remark 4.3. When $q(s)=c s$ for some $c>0$, compactness of positive trajectories in the energy space is satisfied as a special case of the classical theorem of Webb [22]. Indeed then the equation

$$
u_{t t}-u_{x x}+c a(x)\left(u_{t}\right)=0 \quad \text { on } \quad \mathbb{R}^{+} \times(0, L) ; \quad u(t, 0)=u(t, L) \quad \text { on } \quad \mathbb{R}^{+}
$$

generates an exponentially damped linear semi-group in $V \times H$ and the Nemytskii operator $u \rightarrow g(u)$ is compact $V \rightarrow H$.

Remark 4.4. The method of proof of Theorems 2.1 and 2.2 applies also to the more qeneral case of the equation

$$
u_{t t}+A u+Q\left(t, u_{t}\right)=0 \quad \text { on } \quad \mathbb{R}^{+}
$$

where $Q\left(t, u_{t}\right)$ is realized in the form

with

$$
a(t, y) q\left(t, y, u_{t}\right)
$$

$$
\inf _{y \in \omega, t \geq 0} a(t, y)>0
$$

when $q$ satisfies the uniform conditions

$$
\forall \varepsilon>0, \quad \inf _{s \geq \varepsilon, y \in \omega, t \geq 0} q(t, y, s)>0
$$

and

$$
\lim _{\varepsilon \rightarrow 0} \sup _{|s| \leq \varepsilon, y \in \omega, t \geq 0}|q(t, y, s)|=0 .
$$


This is in particular applicable to the problems

$$
u_{t t}-\Delta u+a(x) \tilde{q}\left(x, \nabla u, u_{t}\right)=0 \quad \text { on } \quad \mathbb{R}^{+} \times \Omega ; \quad u(t, x)=0 \quad \text { on } \quad \mathbb{R}^{+} \times \partial \Omega
$$

and

with

$$
u_{t t}-\Delta u=0 \quad \text { on } \quad \mathbb{R}^{+} \times \Omega ; \quad \frac{\partial u(t, x)}{\partial \nu}+a(x) \tilde{q}\left(x, \nabla u, u_{t}\right)=0 \quad \text { on } \quad \mathbb{R}^{+} \times \partial \Omega
$$

$$
\tilde{q}\left(x, \nabla u, u_{t}\right)=\tilde{q}\left(y, \nabla u(t, y), u_{t}(t, y)\right)=: q\left(t, y, u_{t}\right) .
$$

In this case we recover some recent results of Vancostenoble [21] which generalize Slemrod [19].

The author is grateful to the referees for their remarks which led to a useful reorganization of this paper.

\section{REFERENCES}

[1] L. Amerio and G. Prouse, Abstract almost periodic functions and functional equations. Van Nostrand, New-York (1971).

[2] J.M. Ball and M. Slemrod, Feedback stabilization of distributed semilinear control systems. Appl. Math. Optim. 5 (1979) $169-179$.

[3] M. Biroli, Sur les solutions bornées et presque périodiques des équations et inéquations d'évolution. Ann. Math. Pura Appl. 93 (1972) 1-79.

[4] T. Cazenave and A. Haraux, Propriétés oscillatoires des solutions de certaines équations des ondes semi-linéaires. $C$. $R$. Acad. Sci. Paris Sér. I Math. 298 (1984) 449-452.

[5] T. Cazenave and A. Haraux, Oscillatory phenomena associated to semilinear wave equations in one spatial dimension. Trans. Amer. Math. Soc. 300 (1987) 207-233.

[6] T. Cazenave and A. Haraux, Some oscillatory properties of the wave equation in several space dimensions. J. Funct. Anal. 76 (1988) 87-109.

[7] T. Cazenave, A. Haraux and F.B. Weissler, Une équation des ondes complètement intégrable avec non-linéarité homogène de degré 3. C. R. Acad. Sci. Paris Sér. I Math. 313 (1991) 237-241.

[8] T. Cazenave, A. Haraux and F.B. Weissler, A class of nonlinear completely integrable abstract wave equations. J. Dynam. Differential Equations 5 (1993) 129-154.

[9] T. Cazenave, A. Haraux and F.B. Weissler, Detailed asymptotics for a convex hamiltonian system with two degrees of freedom. J. Dynam. Differential Equations 5 (1993) 155-187.

[10] F. Conrad and M. Pierre, Stabilization of second order evolution equations by unbounded nonlinear feedbacks. Ann. Inst. H. Poincaré Anal. Non Linéaire 11 (1994) 485-515.

[11] A. Haraux, Comportement à l'infini pour une équation des ondes non linéaire dissipative. C. R. Acad. Sci. Paris Sér. I Math. 287 (1978) 507-509.

[12] A. Haraux, Comportement à l'infini pour certains systèmes dissipatifs non linéaires. Proc. Roy. Soc. Edinburgh Ser. A 84 (1979) 213-234.

[13] A. Haraux, Stabilization of trajectories for some weakly damped hyperbolic equations. J. Differential Equations 59 (1985) $145-154$.

[14] A. Haraux and V. Komornik, Oscillations of anharmonic Fourier series and the wave equation. Rev. Mat. Iberoamericana 1 (1985) 57-77.

[15] A. Haraux, Semi-linear hyperbolic problems in bounded domains, Mathematical Reports Vol. 3, Part 1 , edited by J. Dieudonné. Harwood Academic Publishers, Gordon \& Breach (1987).

[16] A. Haraux, Systèmes dynamiques dissipatifs et applications, R.M.A. 17, edited by Ph. Ciarlet and J.L. Lions. Masson, Paris (1990).

[17] A. Haraux, Strong oscillatory behavior of solutions to some second order evolution equations, Publication du Laboratoire d'Analyse Numérique 94033, 10 p.

[18] B.M. Levitan and V.V. Zhikov, Almost periodic functions and differential equations. Cambridge University Press, Cambridge (1982).

[19] M. Slemrod, Weak asymptotic decay via a relaxed invariance principle for a wave equation with nonlinear, nonmonotone damping. Proc. Roy. Soc. Edinburgh Ser. A 113 (1989) 87-97.

[20] J. Vancostenoble, Weak asymptotic stability of second order evolution equations by nonlinear and nonmonotone feedbacks. SIAM J. Math. Anal. 30 (1998) 140-154.

[21] J. Vancostenoble, Weak asymptotic decay for a wave equation with weak nonmonotone damping, 17p (to appear).

[22] G.F. Webb, Compactness of trajectories of dynamical systems in infinite dimensional spaces. Proc. Roy. Soc. Edinburgh Ser. A 84 (1979) 19-34. 